\title{
Epidemiological Profile of Patients with Hepatocellular Carcinoma
}

\author{
Auzelivia Pastora Rego Medeiros Falcão ${ }^{1}$, Carlos Henrique de Almeida Cipriano², Guilherme \\ Castro de Queiroz ${ }^{2}$, Nikolas do Vale Pedreira ${ }^{2}$, Amália Cinhtia Meneses Rêgo ${ }^{3}$ and Irami Araújo- \\ Filho $^{3 *}$ \\ ${ }^{1}$ Department of Gastroenterologist and Hepatologist, Brazil \\ ${ }^{2}$ Undergraduate Student of Medicine, Brazil \\ ${ }^{3}$ Postgraduate Program in Biotechnology, Brazil
}

*Corresponding author: Irami Araújo-Filho, Department of Surgery, Potiguar University, Brazil.

To Cite This Article: Irami Araújo-Filho, Epidemiological Profile of Patients with Hepatocellular Carcinoma. 2020 - 7(5). AJBSR.MS.ID.001185. DOI: 10.34297/AJBSR.2020.07.001185.

Received: 阱 February 20,2020; Published: 制 February 27, 2020

\begin{abstract}
Hepatocellular carcinoma (HCC) is the sixth most common cause of cancer in the world, with more than 740,000 cases/year. Hepatocellular carcinoma is related to chronic liver diseases (hepatitis B, C, cirrhosis). Treatment of HCC is variable and may or may not be successful. After treatment, the patient is followed up to prevent and diagnose recurrences. This observational, retrospective and cross-sectional study was carried out at Hospital Universitário Onofre Lopes-HUOL, State of Rio Grande do Norte/Brazil. The epidemiological profile of HCC patients followed up at the Liver Study Center (LSC)-Reference Center in Hepatology was analyzed. Data were obtained from the analysis of medical records from 1995 to 2018, after approval by the Institutional Research Ethics Committee of the 79 patients with confirmed HCC, a male prevalence of $68 \%$ was observed; 56\% of cases affected patients over 60 years of age, and in $49 \%$ of patients, HCC was diagnosed exclusively by imaging method. Concomitant cirrhosis was observed in $63 \%$ of the cases studied. During the 13 years analyzed, the loss of follow-up was $64 \%$, which impacted the quality of care provided to patients at the service. Only $13 \%$ of patients had access to surgical treatment for HCC, and $53 \%$ of cases had less than one year of evolution. The creation of a specialized HCC service would reduce the rates of late diagnosis, loss of follow-up, waiting time for treatment with a positive impact on the prognosis, and increase in survival rates.
\end{abstract}

Keywords: Hepatocellular Carcinoma; Epidemiology; Neoplasms; Liver; Cancer Treatment Protocols; Follow-Up Study

Abbreviations: HCC: Hepatocellular Carcinoma; LSC: Liver Study Center; CT: Computed Tomography; EASL: European Association for the Study of the Liver; MRI: Magnetic Resonance Imaging

\section{Introduction}

Hepatocellular carcinoma (HCC) is cancer arising from hepatocytes, due to genetic mutations that cause these cells to multiply in disorder. This multiplication can be caused by an external agent (hepatitis B and C) or excessive cell multiplication (chronic hepatitis B regeneration) [1,2]. Currently, HCC is responsible for $70-85 \%$ of primary liver neoplasms and the third most common cause of death from cancer. Hepatocellular carcinoma is treated by surgical resection, liver transplantation, radiofrequency ablation, cryoablation and radiotherapy associated or not with chemotherapy [3]. Surgical approaches depend on the size of the tumor and have high rates of recurrence, with a 5-year survival rate of $31 \%$. Radiotherapy is used to treat primary and/ or metastatic HCC, injecting unique microspheres (90Y) directly into primary tumors or infusing the $131 \mathrm{I}$ arterial lipiodol [4-6]. Chemotherapy is reserved for those cases without indications for resection, percutaneous injection of ethanol, acetic acid or arterial embolization [7].

In patients with distant metastases or with portal tumor thrombosis and low functional liver reserve, chemotherapy regimens containing fluorouracil, mitoxartrone, and cisplatin 
are used $[3,8]$. Palliative care improves the quality of life through the prevention and relief of suffering in patients with advanced disease, without any other therapeutic option [9]. The effectiveness of available treatments is related to the adequate follow-up of patients after the procedure. Appropriate monitoring is crucial for the prevention and early diagnosis of recurrences $[6,10]$. Based on this scenario, the present study analyzed the epidemiological profile and follow-up of patients who underwent treatment of hepatocellular carcinoma, taking into account the clinical evolution and the application of current management protocols for patients with HCC.

\section{Methods}

An observational, cross-sectional, and retrospective study in which the epidemiological profile and follow-up of patients with HCC were evaluated. A total of 13,500 records were consulted from the Liver Studies Center at Hospital Universitário Onofre Lopes (HUOL)/Federal University of Rio Grande do Norte (UFRN)/NATAL/ BRAZIL. The Liver Studies Center is a reference center in Hepatology for the entire State of Rio Grande do Norte and neighboring regions. The evaluated period corresponded to the interval between the years 1995-2018. All patients with hepatocellular carcinoma were selected and the epidemiological profile and post-treatment followup were analyzed. The selection of medical records was made by two researchers independently, with a third consultant in case of disagreement regarding the inclusion or not of the selected patient. The study was approved by the Ethics and Research Committee of HUOL on December 10, 2018, under Protocol-CAAE 02068918.2.0000.5292/Plataforma Brasil/Ministry of Health. The general guidelines of the Ministry of Health (MS)/Brazil and the European Association for the Study of the Liver (EASL) were used to define patients with HCC. The diagnosis was made by computed tomography (CT), magnetic resonance imaging (MRI), or ultrasound with contrast and confirmed by biopsy/histopathology. The variables analyzed were sex, age, origin, presence of cirrhosis, proposed treatment, and diagnostic examination of HCC. Data were collected from $04 / 02 / 2019$ to $07 / 31 / 2019$. Data analysis was performed using descriptive statistics using Microsoft Excel@ 2016, with the calculation of percentage, average, median, and standard deviation. The results were translated into the form of graphics for better interpretation. Medical records that contained illegible and doubtful information regarding the patient and his therapeutic management were excluded from the study.

\section{Results and Discussion}

After analyzing the medical records, 79 patients with a confirmed diagnosis of HCC were selected. Of the 79 patients, there was a higher prevalence in males (68\%) (Figure 1). According to world epidemiology, in areas endemic for the disease, the predominance of males is more pronounced due to a higher rate of chronic infection by the hepatitis B virus, alcoholism, higher testosterone, and lower estrogen $[11,12]$. The analysis by age group showed that most patients were over 60 years old, 45 cases (56\%). In Brazil, the average age at diagnosis of 59 years was observed, despite the prevalence in our study is quite significant in patients between 30 59 years old with 33 cases (42\%) and reduced in patients under 30 years old, one claim (1\%) (Figure 2) [6,13]. In patients below 60 years of age, there were comorbidities considered a risk factor for the development of HCC, such as Hepatitis C and alcoholic cirrhosis $[8,14]$.

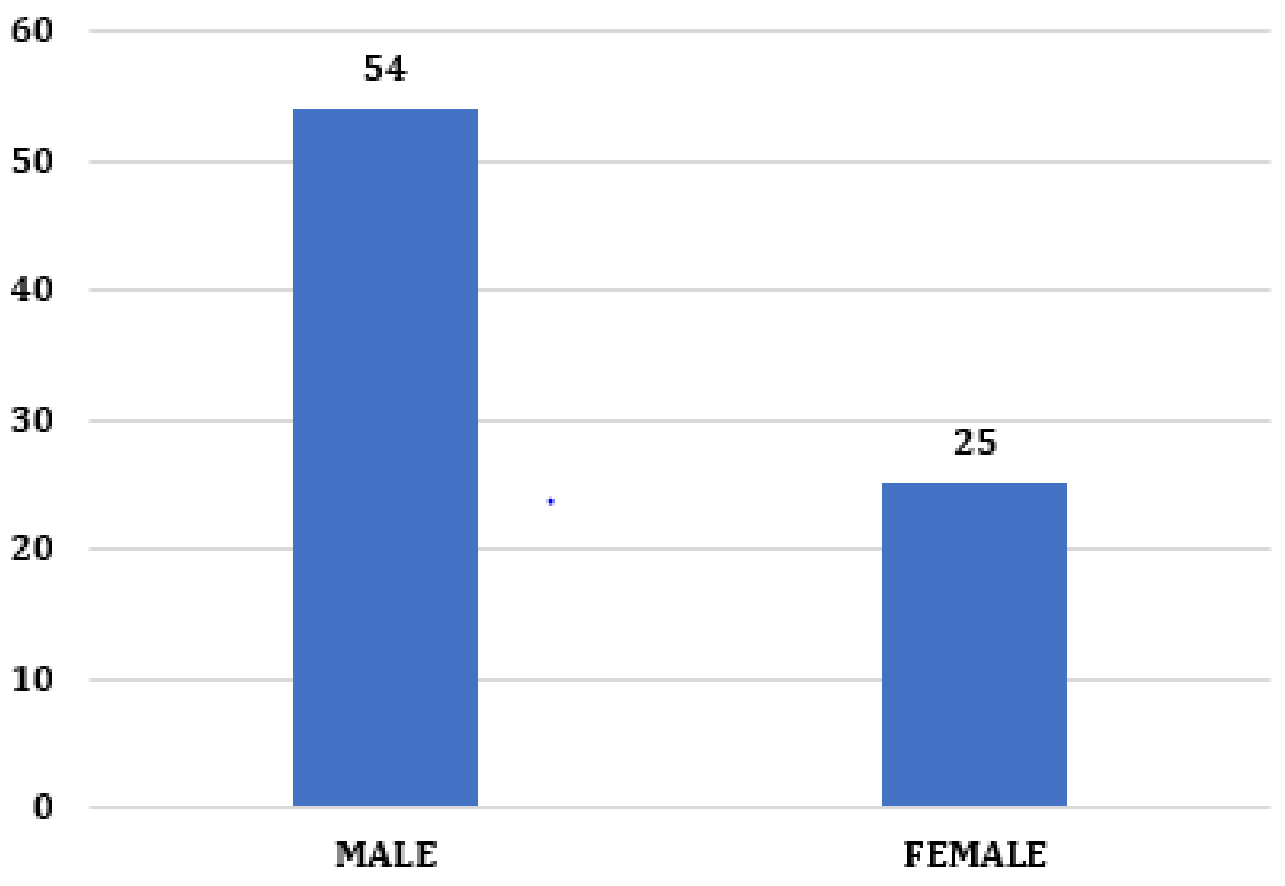

Figure 1: Prevalence of HCC/Gender. 


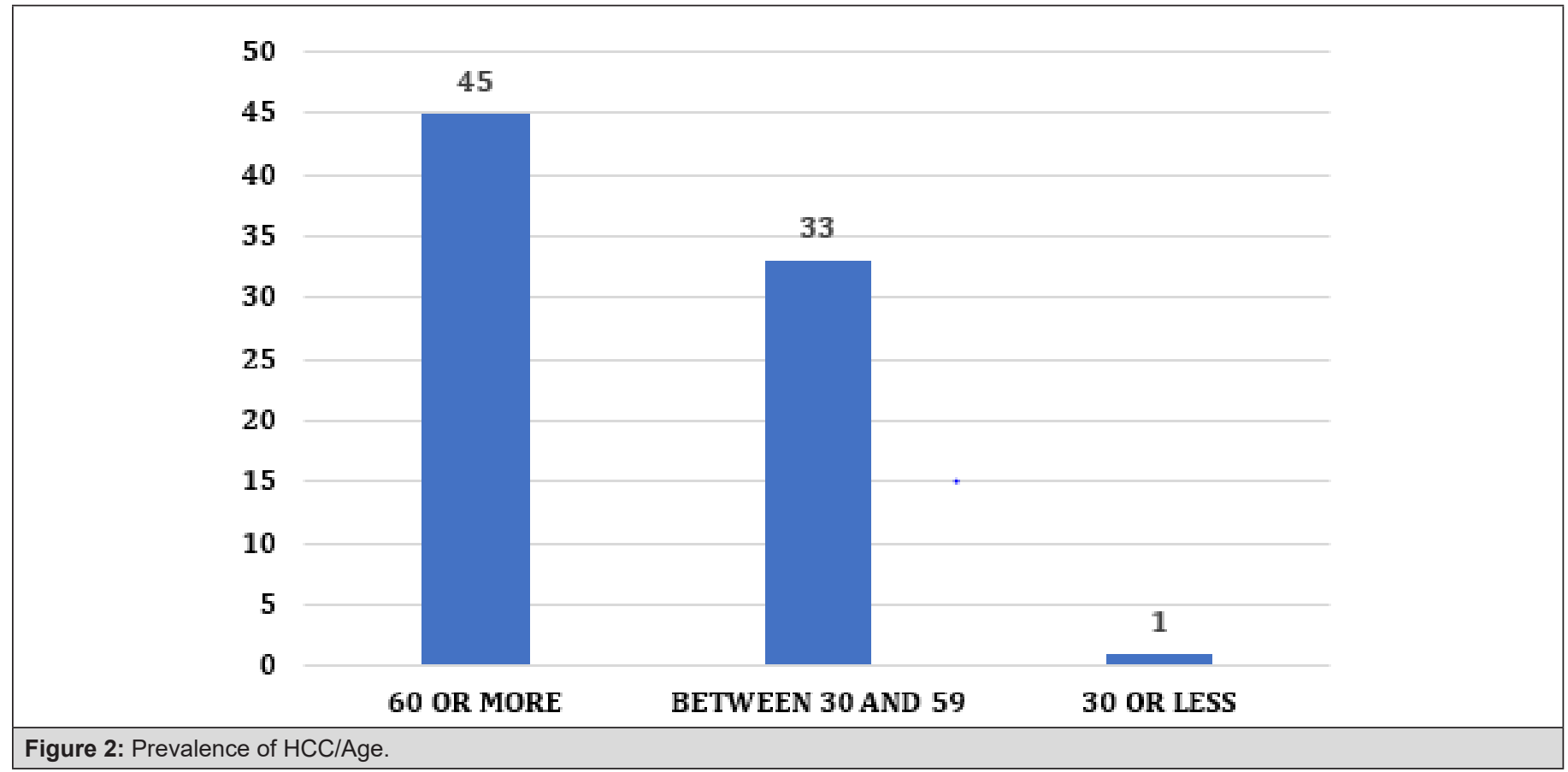

In patients over 60 years of age, age was observed as the primary epidemiological variable for HCC, since the incidence of hepatocellular carcinoma grows progressively with life expectancy in all populations, reaching a peak around 70 years of age [15-17]. Regarding origin, in the metropolitan region of Natal, 43 (54\%) cases were observed, and in the other areas, 36 (46\%) cases (Figure $3)$. The most significant number of patients from the metropolitan region occurred because it is made up of the capital and 15 more cities located around it. Less than $30 \%$ of HCC cases are diagnosed early to allow surgical resection or even liver transplantation [1416]. Thus, early diagnosis is essential for a good prognosis and increased survival of patients with HCC. Currently, the diagnosis can be made through imaging exams, tumor markers, and anatomopathological [3-5,18].

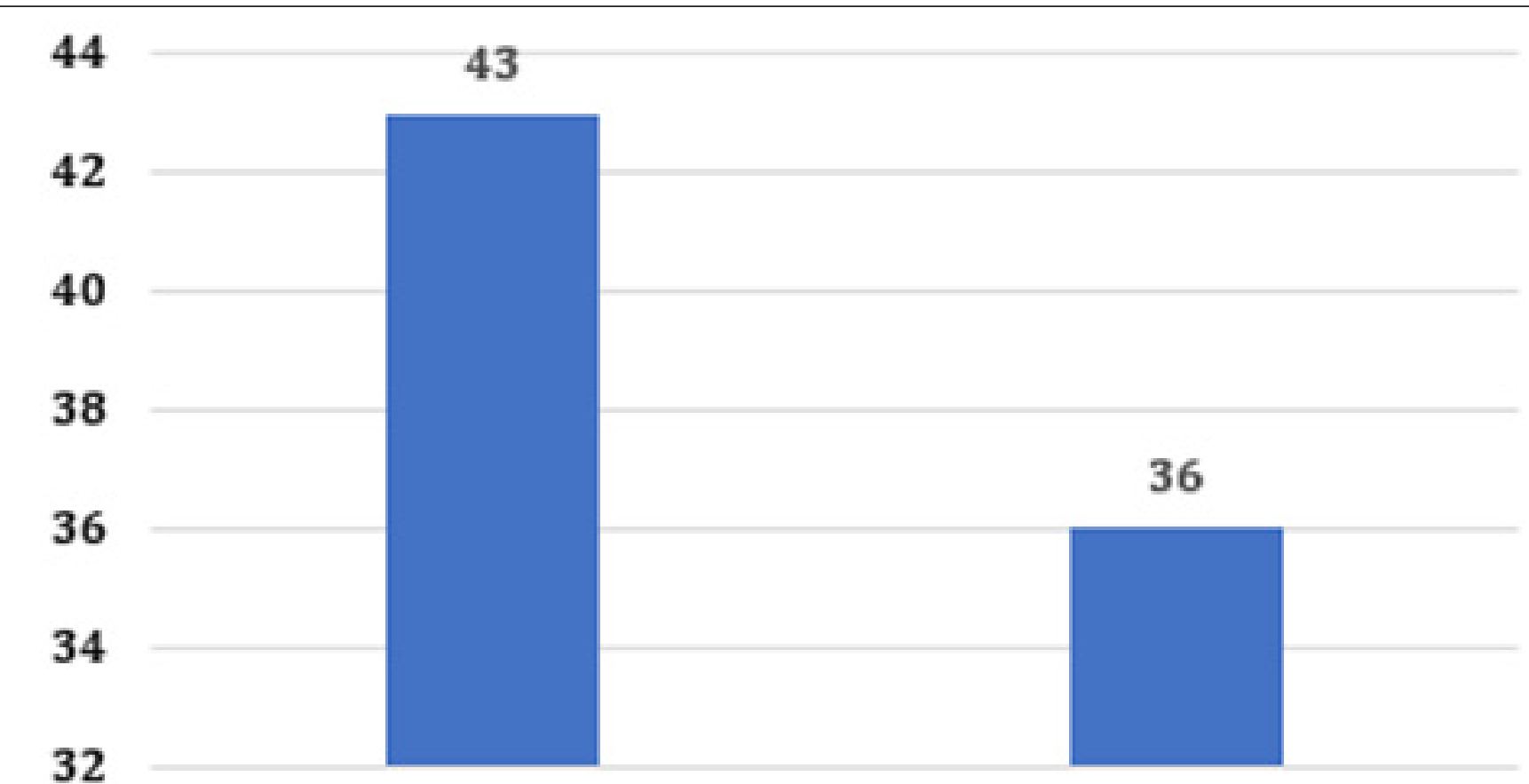


Still, tumor markers are not always increased, and biopsy may not be possible, which makes imaging methods necessary means of radiological diagnosis of the patient with Hepatocarcinoma [19]. In the present study, it was observed that 39 (49\%) cases were diagnosed using imaging methods (MRI, USG, or CT) exclusively. In 20 cases (25\%) alpha-fetoprotein was used in association with radiology to aid in diagnosis; in 13 (16\%) cases, surgical methods (biopsy and paracentesis) were performed; and in 8 (10\%) cases, this data was not included in the medical record (Figure 4). This reveals the importance of imaging tests in the diagnosis and prognosis of this pathology $[15,19]$. In Figure 5, it is possible to observe that considering the presence of cirrhosis, most of the patients analyzed had liver cirrhosis, with 50 cases $(63 \%)$ of etiologies, both alcoholic and Hepatitis C or B and that 29 cases (36\%) did not. have cirrhosis [1,20]. This demonstrates that previous cirrhosis is hugely impactful in cases of HCC. The main risk factors for HCC include infection with HVB and HVC, alcoholic cirrhosis, exposure to aflatoxins, and, mainly, non-alcoholic liver steatosis [2-4,21]. Among the 79 charts analyzed, there were 51 losses from follow-up (64\%). This fact was observed both in the oldest and most recent medical records, which demonstrates the limitation found in this type of study. The causes were several, ranging from the patient's failure to attend the service, such as the lack of registration by the professionals or even the death of the patients included in the study.
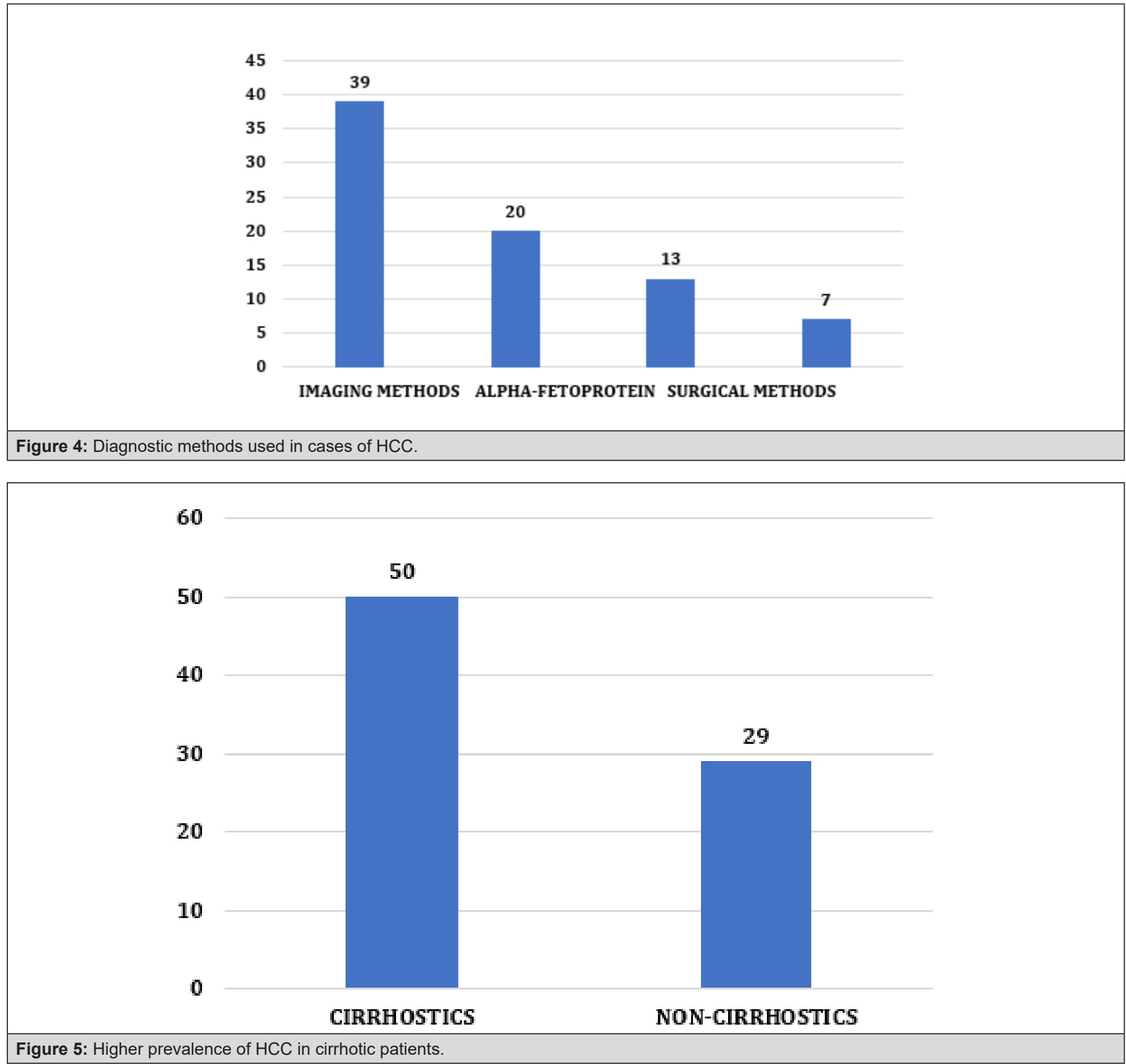
All of this influenced the prognosis of patients with HCC, as it hindered a more in-depth analysis of the sample population [9]. Both timely therapeutic intervention and patient follow-up are essential for good progress, as the follow-up time has a direct impact on the stage at which the HCC is located and the chances of a good prognosis associated with increased survival [5-7]. Another critical point is the time of evolution of these patients. Among the cases analyzed, 42 (53\%) had less than one year of development recorded in the medical records, 23 (29\%) more than one year and $14(17 \%)$ cases, and this data was not included in the medical history (Figure 6). For the collection of this data, the time between the moment of diagnosis and the outcome or loss of segment was taken into account. In general, without intervention, HCC usually evolves with intra and extrahepatic metastases. In these cases, death usually occurs in an average of 10 months caused by cachexia, bleeding from esophageal or gastric varices, and liver failure $[10,22]$.

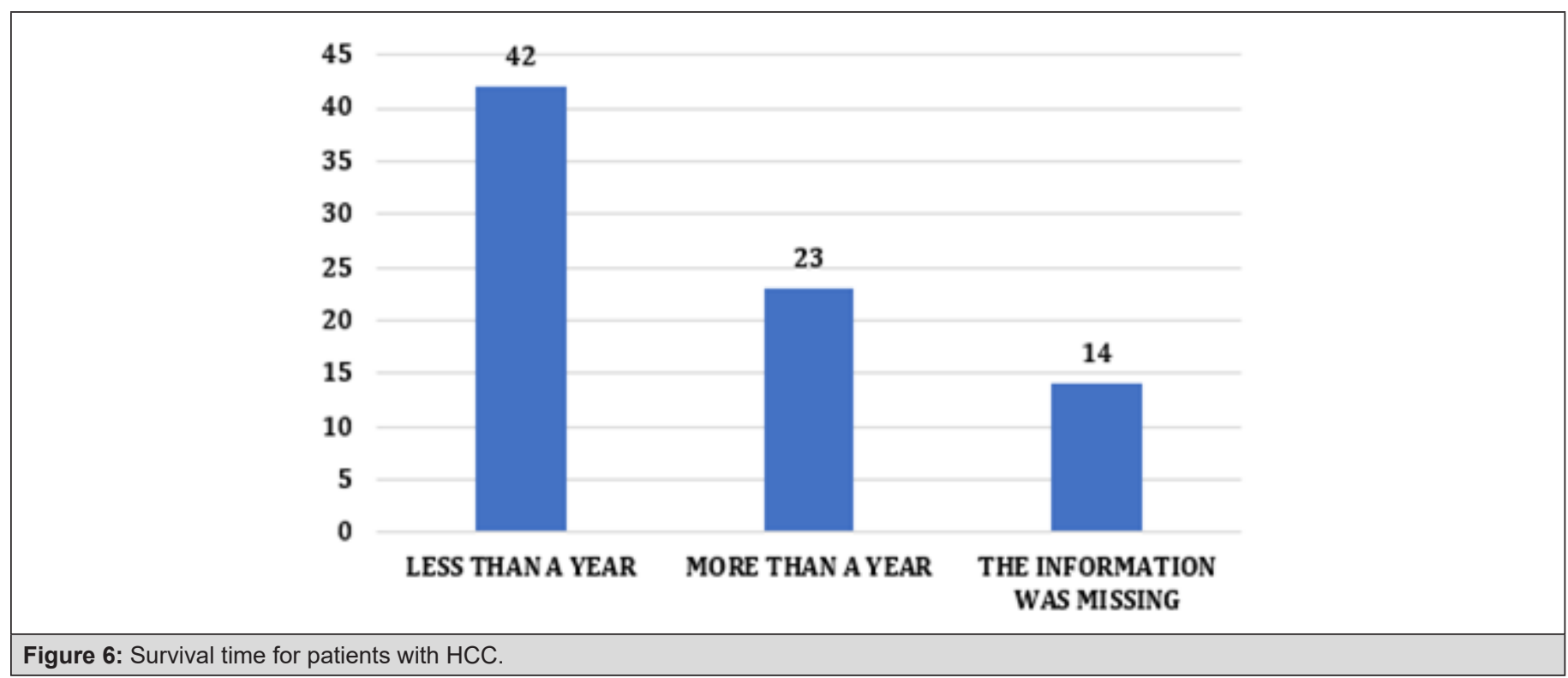

Regarding the treatment of HCC, the therapeutic option depending on the stage at which the tumor was diagnosed and on the presence or not of associated diseases. Surgical treatment, potentially curative, was adopted in the earliest stages of the disease [22-24]. For more advanced stages, medications by chemoembolization or the use of sorafenib aimed to reduce the size of the tumor and inhibit the evolution of the disease [1-3]. Regarding treatment, 22 (26\%) cases underwent chemotherapy, 11 (13\%) surgery for resection or transplantation, 2 (2\%) radioablation, 9 (11\%) palliative methods and $35(44 \%)$ of the cases the type of treatment was not on the chart (Figure 7) [25].

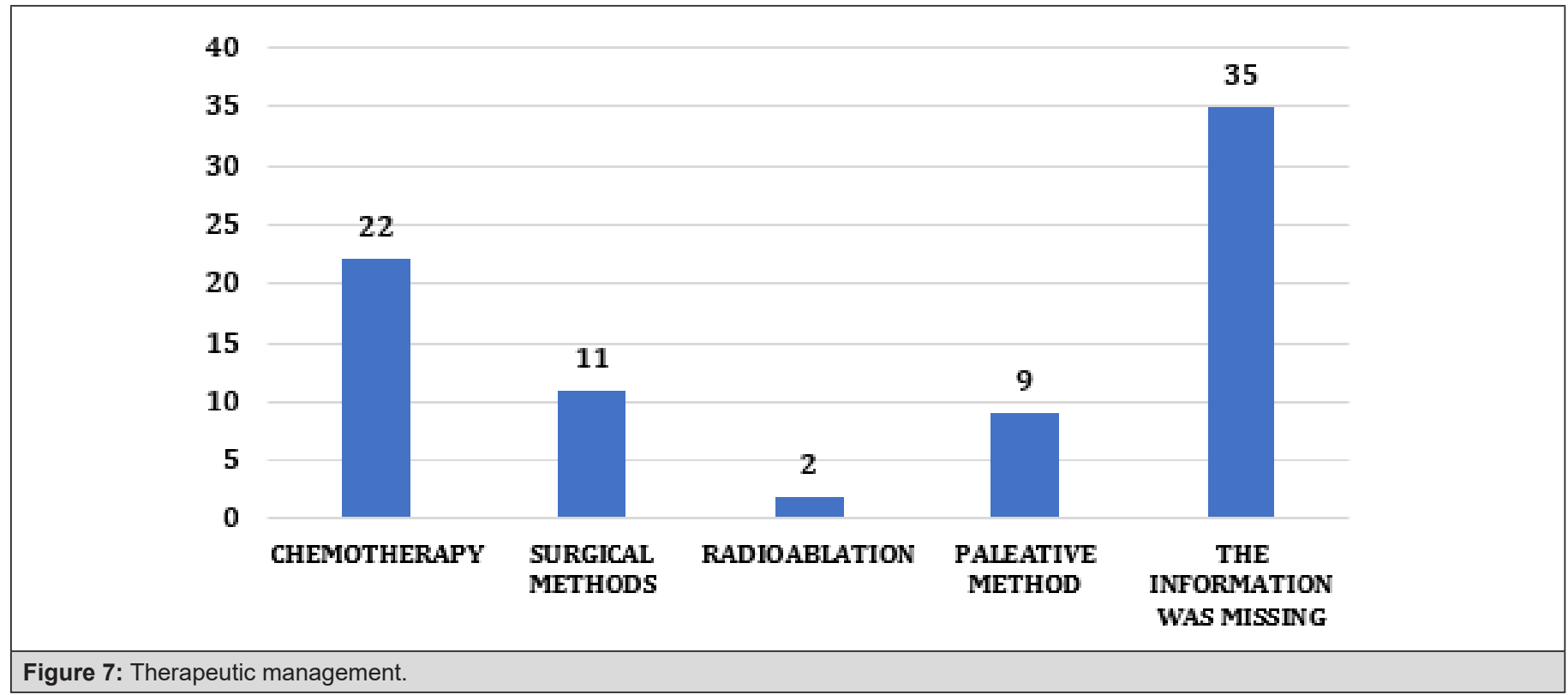


Analyzing these data, we observed that conservative treatments were more used than surgical ones, which could be explained by the fact that in most cases, HCC was diagnosed late, which limited the possibility of a potentially curative surgical intervention [26]. Another point observed in this study was the outcome of the cases analyzed. HCC is very aggressive, with high mortality after the onset of symptoms, most commonly jaundice and ascites [25-27]. If it is detected only in the symptomatic phase, the patient without treatment has an average life expectancy of less than one month, and even at this stage, the available treatments are limited and ineffective [26-28]. Among the medical records observed, 8 (10\%) cases had confirmed death; 7 (9\%) disease remission and 64 (81\%) cases had no recorded disease outcome (Figure 8).The limitations of the study were the high percentage of patients diagnosed with HCC who had segment loss, which made it difficult to interpret data as an outcome and treatment used associated with the lack of information in the medical records about how patients were treated at the LSC service observed mainly in the oldest medical records.

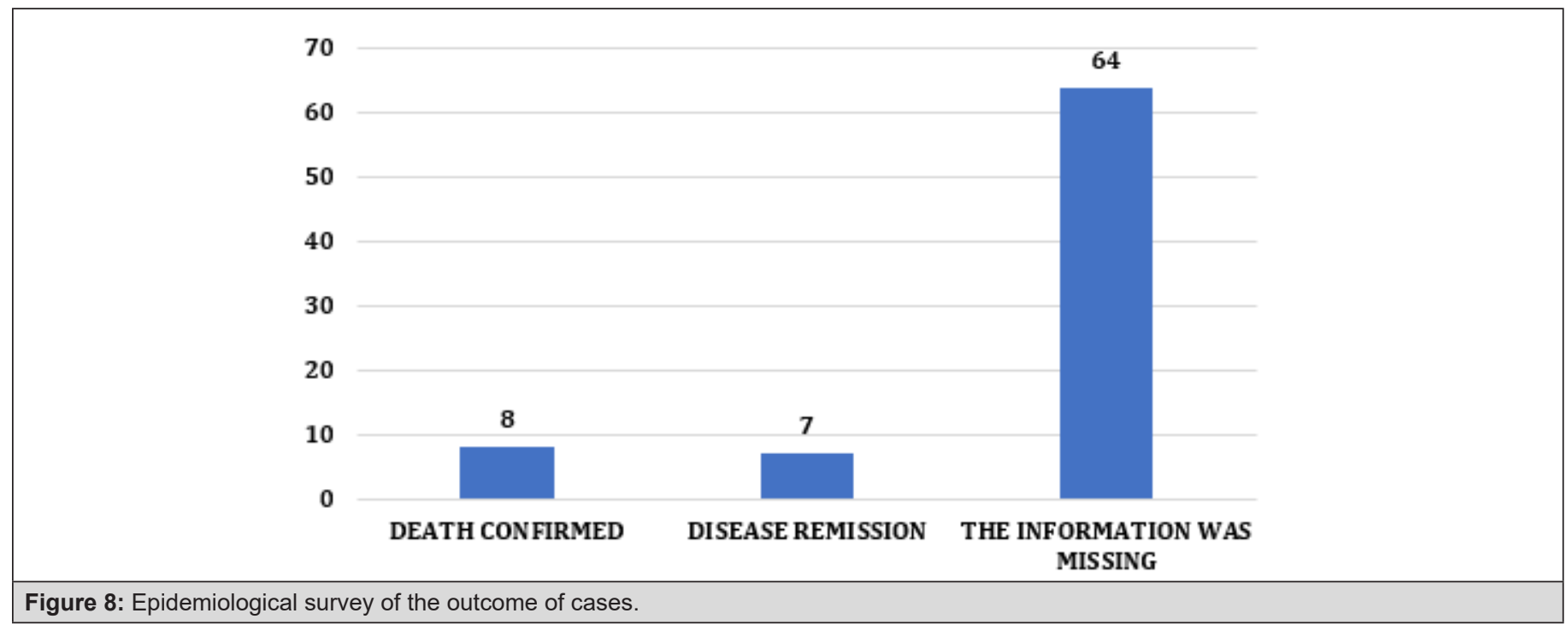

\section{Conclusion}

The high mortality from hepatocellular carcinoma is related to the late diagnosis of the disease. Most cases are diagnosed in advanced stages, where surgical interventions are no longer indicated. The prevalence of the disease, especially in patients under 50 years of age, is related to liver cirrhosis and grows in proportion to the increase in life expectancy. Therefore, the need for more effective screening of the disease is essential for a good prognosis for patients, especially those in the risk factor groups evidenced in this study: male patients, over 50 years old, with hepatitis B, C and cirrhotics. The loss of follow-up in $64 \%$ of the cases analyzed was another finding in patients treated by the LSC. This phenomenon harms the prognosis of these patients, as it makes it challenging to optimize the treatment of patients with HCC and increases the chances of a poor prognosis. An outpatient clinic specialized only in HCC would be a useful measure in combating loss of follow up and late diagnosis in these individuals. The reference to the service would be facilitated, which would reduce the waiting time for treatment with a positive impact on the prognosis, reduction in segment loss and an increase in survival rates.

\section{Compliance with Ethical Standards}

\section{Acknowledgments}

The authors thank the Ph.D. in Health Sciences and Teaching and Research Manager at League Against Cancer, Profa. Dra. Amália
Rêgo, for her contribution and relevance to the scientific discussion and supervision of this research, acting as an expert consultant on the bibliographic survey, analysis, and scientific advice. We also thank all the study components for their dedication and effort to build a scientifically validated quality study.

\section{Disclosure of Conflict of Interest}

There are no conflicts of interest to declare by any of the authors of this study.

\section{References}

1. Armengol C, Sarrias MR, Sala M (2018) Hepatocellular carcinoma: present and future. Med Clin 150(10): 390-397.

2. Bruix J, Sherman M (2011) Management of hepatocellular carcinoma: an update. Hepatology 53(3):1020-1022.

3. Chedid MF, Kruel CRP, Pinto MA, Grezzana-Filho TJM, Leipnitz I, et al. (2017) Hepatocellular carcinoma: diagnosis and operative Management. Arq Bras Cir Dig 30(4):272-278.

4. Conte VP (2000) Hepatocellular carcinoma. Arq Gastroenterol 37(2): 133-143.

5. (2018) European Association for the Study of the Liver. EASL Clinical Practice Guidelines: Management of hepatocellular carcinoma. J Hepatol 69(1):182-236.

6. Gomes MA, Priolli DG, Tralhão JG, Botelho MF (1992) Hepatocellular carcinoma: epidemiology, biology, diagnosis, and therapies. Rev Assoc Med Bras 59(5): 514-524.

7. González-Huezo MS (2013) Hepatocellular carcinoma. Rev Gastroenterol Mex 78(1): 108-110. 
8. Hermes HR, Lamarca IC (2013) Palliative care: an approach based on the professional health categories. Cien Saude Colet 18(9): 2577-2588.

9. De Lope CR, Tremosini S, Forner A, Reig M, Bruix J (2012) Management of HCC. J Hepatol 56(1): 75-87.

10. Ayuso C, Rimola J, Vilana R, Burrel M, Darnell A (2018) Diagnosis and staging of hepatocellular carcinoma (HCC): current guidelines. Eur J Radiol 101: 72-81.

11. Ozer Etik D, Suna N, Boyacioglu AS (2017) Management of hepatocellular carcinoma: prevention, surveillance, diagnosis, and staging. Exp clin transplant 15(2): 31-35.

12. Bruix J, Reig M, Sherman M (2016) Evidence-based diagnosis, staging and treatment of patients with hepatocellular carcinoma. Gastroenterology 150(4): 835-853.

13. Bellissimo F, Pinzone MR, Cacopardo B, Nunnari G (2015) Diagnostic and therapeutic management of hepatocellular carcinoma. World Gastroenterol 21(42): 12003-120021.

14. Schlachterman A, Craft WW, Hilgenfeldt E, Mitra A, Cabrera R (2015) Current and future treatments for hepatocellular carcinoma. World J Gastroenterol 21(28): 8478-8491.

15. Lee YJ, Lee JM, Lee JS, Lee HY, Park BH (2015) Hepatocellular carcinoma: diagnostic performance of multidetector CT and MR imaging-a systematic review and meta-analysis. Radiology 275(1): 97-109.

16. Mazzaferro V, Regalia E, Doci R, Andreola S, Pulvirenti A, et al. (1996) Liver transplantation for the treatment of small hepatocellular carcinomas in patients with cirrhosis. N Engl J Med 334(11): 693-699.

17. Ono T, Yamanoi A, Nazmy El Assal O, Kohno H, Nagasue N (2001) Adjuvant chemotherapy after resection of hepatocellular carcinoma causes deterioration of long-term prognosis in cirrhotic patients: metaanalysis of three randomized controlled trials. Cancer 91(12): 2378-2385.

18. El Jabbour T, Lagana SM, Lee H (2019) Update on hepatocellular carcinoma: Pathologist's review. World J Gastroenterol 25(14): 1653 1665.
19. Rastogi A (2018) Changing role of histopathology in the diagnosis and management of hepatocellular carcinoma. World J Gastroenterol 24(35): 4000-4013

20. Kim HY, Kim W, Jung YJ, Lee JH, Yu SJ, et al. (2018) A Model for Adaptive Decision Making of "Ablate-and-Wait" Versus Transplantation in Patients With Hepatocellular Carcinoma. J Clin Gastroenterol 52(7): 655-661.

21. Cadier B, Bulsei J, Nahon P, Seror O, Laurent A, et al. (2017) Early detection and curative treatment of hepatocellular carcinoma: A costeffectiveness analysis in France and in the United States. Hepatology 1237-1248.

22. Ramanathan R, Sharma A, Lee DD, Behnke M, Bornstein K, et al. (2014) Multimodality therapy and liver transplantation for hepatocellular carcinoma: a 14-year prospective analysis of outcomes. Transplantation 98(1): 100-106.

23. Caldwell S, Park SH (2009) The epidemiology of hepatocellular cancer: from the perspectives of public health problem to tumor biology. J Gastroenterol 44(19): 96-101.

24. Bangaru S, Marrero JA, Singal AG (2020) Review Article: New Therapeutic interventions for advanced hepatocellular carcinoma. Aliment Pharmacol Ther 51(1): 78-89.

25. Bouattour M, Mehta N, He AR, Cohen EI, Nault JC (2019) Systemic treatment for advanced hepatocellular carcinoma. Liver Cancer 8(5): 341-358.

26. Gbolahan OB, Schacht MA, Beckley EW, LaRoche TP, O’Neil BH, et al. (2017) Locoregional and systemic therapy for hepatocellular carcinoma. J Gastrointest Oncol 8(2): 215-228.

27. Medavaram S, Zhang Y (2018) Emerging therapies in advanced hepatocelular carcinoma. Exp Hematol Oncol 7:17.

28. Lurje I, Czigany Z, Bednarsch J, Roderburg C, Isfort P, et al. (2019) Treatment strategies for hepatocellular carcinoma-a multidisciplinary approach. Int J Mol Sci 20(6). 